\title{
RETRACTED ARTICLE: A reusable aptasensor based on the dual signal amplification of Ce@AuNRs-PAMAM-Fc and DNA walker for ultrasensitive detection of TNF- $a$
}

\author{
Yu Ding ${ }^{1} \cdot$ Ming Zhang ${ }^{2} \cdot$ Cunyu $\mathrm{Li}^{1} \cdot$ Bin Xie ${ }^{1} \cdot$ Guiping Zhao ${ }^{2} \cdot$ Yingying Sun $^{1}$ (D) \\ Received: 22 September 2020 / Revised: 24 November 2020 / Accepted: 6 December 2020 / Published online: 6 January 2021 \\ (C) The Author(s), under exclusive licence to Springer-Verlag GmbH, DE part of Springer Nature 2021
}

The authors have retracted this article. After publication the authors found that when using the aptasensor they were unable to replicate the experimental results reported. The authors therefore no longer have confidence in the conclusions of the article. All authors agree to this retraction. The online version of this article contains the full text of the retracted article as Supplementary Information.

Supplementary Information The online version contains supplementary material available at https://doi.org/10.1007/s10008-020-04885-8.

1 Tengzhou Central People's Hospital, The Affiliated Tengzhou Hospital of Xuzhou Medical University, Tengzhou 277500, China

2 Department of Clinical Laboratory, Yangzhou Maternal and Child Health Hospital, The Affiliated Hospital of Yangzhou University Medical College, Yangzhou 225000, China 